\title{
Health-related quality of life of patients with a recent fracture attending a fracture liaison service: a 3-year follow-up study
}

\author{
N. $\mathrm{Li}^{1} \cdot$ M. van Oostwaard ${ }^{2,3} \cdot$ J. P. van den Bergh ${ }^{2,3,4} \cdot$ M. Hiligsmann ${ }^{1} \cdot$ A. Boonen ${ }^{5} \cdot$ S. M. J. van Kuijk ${ }^{6} \cdot$ L. Vranken $^{2,3}$. \\ S. P. G. Bours ${ }^{5}$. C. E. Wyers ${ }^{2,3}$
}

Received: 28 June 2021 / Accepted: 8 October 2021 / Published online: 20 October 2021

(c) The Author(s) 2021

\begin{abstract}
Summary This study explored the course of health state utility value over 3 years in patients with a recent fracture attending a Fracture Liaison Service and suggested that the overall change in health-related quality of life was not significant, although significant improvements were observed at 6 and 12 months compared to baseline.

Introduction To estimate the 3-year health-related quality of life (HRQoL) of patients with a recent fracture presenting at a Fracture Liaison Service (FLS) and to explore factors associated with health state utility value (HSUV).

Methods Patients' HSUVs were derived from the EQ-5D-5L and SF-6D and calculated at six time points. Multiple imputation was applied for missing data. Linear mixed-effects regression analysis with random intercept and slope was applied to explore the course of HSUV over 3 years. The impact of subsequent fracture and the length of time between FLS visit and patients' index fracture on HSUV were also investigated. A backward stepwise elimination was applied to identify factors associated with HSUV.

Results A total of 499 patients were included. The change of EQ-5D HSUV was not significant over 3-year follow-up $(P=0.52)$, although slightly but significantly higher HSUV was captured at 6 months (mean difference $(\mathrm{MD}): 0.015, P=0.02)$ and 12 months (MD: 0.018, $P=0.01$ ). There was no significant difference in the course of EQ-5D HSUV between fracture locations $(P=0.86)$. A significant increase in HSUV was only captured for patients had shorter time period ( $<107$ days) between FLS visit and their index fracture. Suffering a subsequent fracture was associated with significant QoL loss (MD: $-0.078, P<0.001)$. Subsequent fracture, previous treatment with anti-osteoporosis medication, a prevalent vertebral fracture (grade 2 or 3), use of a walking aid, previous falls, and higher BMI were negatively associated with mean EQ-5D HSUV over 3 years. Comparable results were found using SF-6D HSUV. The lack of HRQoL data immediately after fracture and selection bias were two main limitations.

Conclusion The 3-year change in HSUV was not statistically significant, although significant improvements were observed at 6 and 12 months in comparison with baseline. Six factors were negatively associated with EQ-5D HSUV.
\end{abstract}

Keywords EQ-5D-5L $\cdot$ Health utility $\cdot$ Longitudinal analysis $\cdot$ SF-6D

N. Li

n.li@maastrichtuniversity.nl

1 Department of Health Services Research, CAPHRI Care and Public Health Research Institute, Maastricht University, P.O. Box 616, 6200 MD Maastricht, The Netherlands

2 Department of Internal Medicine, Medical Centre, VieCuri, Venlo, The Netherlands

3 Department of Internal Medicine and NUTRIM Research Institute, Maastricht University Medical Centre, Maastricht, The Netherlands
4 Faculty of Medicine, Hasselt University, Hasselt, Belgium

5 Department of Internal Medicine, Division of Rheumatology, Maastricht University Medical Centre, and CAPHRI Research Institute, Maastricht University, Maastricht, The Netherlands

6 Department of Clinical Epidemiology and Medical Technology Assessment, Maastricht University Medical Centre, Maastricht, The Netherlands 


\section{Introduction}

The increasing prevalence of osteoporosis is associated with increased risk of a bone fracture [1]. A Dutch study based on claims data from all Dutch healthcare insurers reported an annual average of 114,116 patients with a fracture was identified between 2009 and 2011, of which $32 \%$ were attributed to osteoporosis [2]. Patients with a recent fracture after the age of 50 years have an increased risk of subsequent fractures. This risk, which is referred to as imminent subsequent fracture risk [3], is highest immediately after the initial fracture and then declines. In addition, the majority of deaths following fractures occur within the first year; thereafter, the excess mortality gradually declines [4]. From the perspective of caregivers, morbidity and mortality following a fracture are important clinical considerations, along with substantial loss of patients' quality of life.

To improve secondary fracture prevention, Fracture Liaison Services (FLSs) are advocated as the most appropriate and effective approach to identify, investigate, and treat patients at risk of new fractures. The first FLS was introduced in 1999 by McLellan and colleagues in the UK [5]; since then, awareness of initiating FLSs worldwide has increased through the Capture the Fracture (CTF) campaign in 2012 by the International Osteoporosis Foundation (IOF) [6] and by other professional organizations [7, 8]. Until May 2021, 644 FLSs (registered in CTF) have been implemented in 48 countries; nearly half of these are in Europe.

To capture the full burden of fractures for a society, it is essential to assess their impact on health. Insight into the loss of healthy life years can facilitate rational decisionmaking when allocating resources across fracture types or diseases [9]. Health state utility value (HSUV) measures are a specified type of health-related quality of life (HRQoL) instruments that reveal the society's preference or value for specific health states. HSUV is an essential component in economic evaluations, used to establish whether the cost of a new intervention can be justified in terms of expected health benefits [10]; it can help decision-makers in prioritizing health interventions.

In fracture research, a large number of studies were conducted to investigate HSUV in patients not attending an FLS. For example, the large ICUROS study [9] reported that fractures resulted in substantial HRQoL loss directly after fracture, and the HRQoL improved after 4 months but did not return to pre-fracture levels. In 2014, a metaanalysis identified 62 studies that reported HSUVs after hip, vertebral, or distal forearm fracture [13]; the study populations were heterogeneous (e.g., pre-fracture, posthip/vertebral/wrist fracture), and most studies had a small sample size and were limited by short follow-up periods, indicating that fracture events were associated with decrements in HSUVs which differed between fracture types.

Very few studies were conducted to investigate HRQoL in patients with fractures attending an FLS [14, 15], especially the course of HSUV in the long term. Therefore, the main objective of this study was to investigate the course of HSUV in patients with a recent fracture presenting at an FLS in the Netherlands, as measured by two generic preferencebased instruments: the EuroQol (EQ-5D-5L) and the Short Form Health Survey (SF-36), over a 3-year follow-up. In addition, considering previous studies $[9,11,16,17]$ indicated that demographics and fracture-related characteristics such as age, previous fracture, hospitalization, and treatment initiation were significantly associated with patients' HSUV, our secondary objective was to identify factors associated with HSUV in patients at the FLS.

\section{Materials and methods}

\section{Subject and study procedures}

This study used data from the "FX MoVie Study," which is a 3-year prospective observational study conducted at the FLS of VieCuri Medical Center in Venlo, the Netherlands [18]. The study protocol (registration number NL45707.072.13) was approved by an independent Medical Ethics Committee and complied with the Declaration of Helsinki. All patients gave written informed consent prior to participation and after receiving oral and written information on the study.

According to standard care, a nurse specialized in osteoporosis invited all patients aged 50 years and older, who visited the emergency department (ED) because of a recent clinical vertebral or non-vertebral fracture, to the FLS. All patients attending the FLS between October 2014 and June 2016 were screened for participation in the "FX MoVie Study." A total of 1380 FLS attenders were screened for eligibility, of whom 990 were eligible to participate and a total of 500 patients aged between 50 and 90 years with a recent, radiologically confirmed fracture participated. We excluded non-Caucasian patients, patients with a fracture due to highenergy trauma, bone metastasis, failure of prosthesis, or osteomyelitis, and patients with cognitive impairment.

All participants received a detailed questionnaire for evaluating clinical risk factors for fractures, such as medical history, medication, previous fractures, and calcium and vitamin D intake, and were scheduled for dual X-ray absorptiometry (DXA) measurement, vertebral fracture assessment (VFA), and a blood test. In addition to the questionnaire for evaluating risk factors, HRQoL questionnaires (EQ-5D-5L and SF-36) were filled out at 3, 6, 12, 24 , and 36 months after inclusion. Of note, the patients' 
first visits at the FLS were scheduled 3-4 months after their fracture, so HRQoL data immediately after their fracture were not available. Three and 6 months after inclusion, patients received the HRQoL questionnaires and a fall diary. Furthermore, they received a telephone call from the research assistant to verify whether they sustained a fall or a subsequent fracture and to complete the questionnaires in case of missing data. At 12, 24, and 36 months after inclusion, patients came to the hospital for a study visit and the questionnaires were repeated.

Bone mineral density (BMD) in the left or right hip and the lumbar spine was determined using DXA with the Hologic QDR 4500 (Hologic, Bedford, MA, USA). Diagnosis of osteoporosis was based on the World Health Organization criteria for BMD [19] according to the lowest value of T-score in femoral neck, total hip, or lumbar spine: osteoporosis as a T-score of -2.5 or less, osteopenia as a T-score between -2.5 and -1.0 , and normal BMD as a T-score of -1.0 or higher.

Assessment of vertebral fractures (VFs) was performed via vertebral fracture assessment (VFA). VFs were graded according to the grading of Genant et al. [20] as mild (grade 1, 20-24\% reduction in vertebral body height at the anterior, mid, or posterior location), moderate (grade $2,25-39 \%$ reduction), or severe (grade $3, \geq 40 \%$ reduction), respectively.

If laboratory results were abnormal, additional investigations were performed for detailed evaluation of newly diagnosed contributors to secondary osteoporosis or other metabolic bone disorders and treatment was initiated when necessary.

\section{Demographics and disease-related characteristics}

The socio-demographics included age (years at time of fracture), gender, and body mass index (BMI). Baseline fracture-related characteristics were collected through (1) questionnaire (and further verified during FLS visit): smoking, fracture site, previous fracture, previous falls (last year), parental hip fracture, use of a walking aid, visual and hearing impairment, previous treatment with anti-osteoporosis medication (AOM), and medical history (which is classified based on International Classification of Diseases version 10), and (2) laboratory tests: BMD, prevalent VFs, secondary osteoporosis, and vitamin D deficiency. In addition, the specific times of new falls and subsequent fractures were recorded for each patient during 3-year follow-up, and we assumed that none of the patients had a fall or a subsequent fracture between their baseline fracture (i.e., the fracture for which they were invited to attend the FLS) and the time they attended the FLS.

\section{Fracture classification}

Patients' index fractures were recorded in electronic Case Report Forms. For purpose of analyses, these fractures were grouped into ten categories according to their location as clavicle/scapula, humerus, radius/ulna, hand/foot, vertebra, rib/sternum, pelvis, femur, tibia/fibula/patella, and multiple fractures (if patients had more than one index fracture). In addition, based on visual inspection, patients with femoral, vertebral, or multiple fractures had a strikingly lower baseline HSUV in comparison with patients with other fractures; the pre-defined ten categories were further divided into two groups (femoral/vertebral/multiple fractures vs. other fractures) to investigate between-group differences.

\section{Health state utility value (outcome)}

HRQoL is expressed in the form of HSUV, which is scored on a scale that assigns a value of 1 to a state equivalent to full health and 0 to a state equivalent to death. In our study, HSUV were calculated according to EQ-5D-5L and SF-36 data. The EQ-5D-5L quantifies health status in terms of five dimensions: mobility, self-care, usual activities, pain/discomfort, and anxiety/depression. Patients score each dimension on a five-level Likert scale (no problem, slight problem, moderate problem, severe problem, and extreme problem). To translate the EQ-5D profiles to societal HSUVs, a value set based on population preferences in the Netherlands was used [21]. The predicted values for the EQ-5D-5L range from -0.446 to 1 , where HSUV below 0 represents health states considered worse than death. The SF-6D was derived from the SF-36 health status measure (version 1, UK Programme) including 6 dimensions of health: physical functioning, social functioning, role limitations, pain, mental health, and vitality, with each dimension having four to six levels. The societal HSUVs were computed using the algorithm developed by Brazier et al. [22]. The predicted values for the SF-6D range from +0.291 to +1 .

\section{Statistical analysis}

For baseline characteristics, descriptives are provided as means and standard deviations (SD) for continuous variables, number, and percentage (\%) for categorical variables. Comparisons between groups (different fracture sites) were conducted using the independent samples $t$-tests or one-way analysis of variance (ANOVA) for continuous variables, and Pearson's chi-square test or Fisher's exact test for categorical variables.

Multiple imputation (MI) with fully conditional specification was employed to impute missing EQ-5D and SF-6D data. The number of imputations was set to 18 , given that approximately $18 \%$ of incomplete cases were identified. 
Patients' missing index values were drawn at six time points, using predictive mean matching. Details of missing data and MI were reported in Supplement 1.

Linear mixed-effects regression with random intercept and slope for patients was applied to compare the mean HSUV between baseline and each time point, to explore the course of the HSUV for all sites of fracture over the 3-year follow-up, and to investigate the impact of fracture site on the course of HSUV over time by including an interaction term of time and baseline fracture group (categorical variable with ten pre-defined categories) in the model.

Additionally, given that the time period between index fracture and FLS visit varied among patients, a subgroup analysis was conducted to explore the difference in the change of HSUV. Since this time period was normally distributed, we used the mean (107 days) as a cut-off to categorize our patients into two groups (i.e., time between index fracture and FLS visit $<107$ days vs. $\geq 107$ days). A clustered line graph was used to visually display the difference in HSUV change over time and the mean difference (MD) was calculated between baseline and each time point. Besides, considering patients' characteristics might influence the results, we also investigated the difference in five most relevant baseline characteristics, i.e., age, gender, $\mathrm{BMD}$, index fracture (femoral/vertebral/multiple fracture, other fractures), and medical history (ICD-10 coded diseases) between two groups using previous mentioned statistical method to further validate our results.

Moreover, the impact of subsequent fracture on HSUV was also investigated. Of note, 20 patients already had a subsequent fracture before the first FLS visit and were therefore excluded for the following analyses. First, the subsequent fracture was treated as a time-varying variable to explore the overall association with HSUV, both between-subjects and within-subjects interpretation were provided. Second, for patients who had a subsequent fracture (during follow-up), the HSUVs before and after a subsequent fracture were compared for these patients. To capture the maximum impact of a subsequent fracture, the HSUV just before and immediately after the subsequent fracture was treated as pre- and postHSUV, respectively (i.e., if one patient had a subsequent fracture at 6 months, the HSUV at 3 months was treated as pre-HSUV and the HSUV at 6 months as post-HSUV). Third, the impact of different locations of subsequent fracture was also investigated. Patients were subdivided into two groups (subsequent femoral/vertebral/multiple fractures vs. subsequent other fractures), the HSUVs before and after a corresponding subsequent fracture were compared for each subgroup. Fourth, we subsequently compared the HSUVs in the group of patients without subsequent fracture to those with a subsequent fracture and applied the median time to subsequent fracture (which was 354 days) to both groups in order to compare HSUVs in the time period before and after subsequent fracture (period 1: 0-364 days vs. period 2: 365-1095 days) in both groups. Given the HSUVs were estimated at discrete time points, and the median time to subsequent fracture was 364 days, the mean HSUV of baseline, 3 months, 6 months, and 12 months was therefore treated as pre-HSUV, and the mean HSUV of 24 months and 36 months was treated as post-HSUV for both groups. Through pre-testing, normal distribution was indicated for the difference (in mean HSUV) between period 1 and 2 for both groups, and given the HSUV of each patient was repeated measured, mean difference was therefore calculated through paired-samples $T$-test.

Furthermore, the identification of factors associated with the average HSUV (over 3 years) was also conducted, using the linear mixed-effect regression model. The abovementioned demographics and disease-related characteristics (16 baseline and two longitudinally assessed variables) were considered potential factors and therefore included in the model. A backward stepwise elimination was applied to omit insignificant factors from the model.

The above-mentioned longitudinal analyses for EQ-5D HSUV were included as the main analysis, and the analyses based on SF-6D HSUV were included as sensitivity analysis. All mixed-effects regression analyses were adjusted for age, gender, and baseline BMD. All analyses were conducted using SPSS (version 26.0, IBM Statistics), and a $P$-value of $\leq 0.05$ was considered statistically significant.

\section{Result}

\section{Baseline characteristics}

After multiple imputations, 499 patients with one or more recent fractures were included in our analyses. Of note, one patient did not complete any questionnaire at all, so no imputation was conducted for this patient, who was therefore excluded from the whole analysis. Baseline characteristics according to fracture site are presented in Table 1. Patients were on average $64.6 \pm 8.6$ years, and $71.3 \%$ were females. One hundred ten $(22.0 \%)$ patients were diagnosed with osteoporosis, 133 (26.7\%) had at least one VF, and 54 (10.8\%) patients reported that they have ever used AOM, at the time of FLS visit, treatment was initiated or continued in 175 (35\%) of patients. More than $90 \%$ of patients had one or more comorbidities (ICD-10 coded disease). On average, patients attended the FLS 107 days after their index fracture.

\section{EQ-5D health state utility value}

The estimated EQ-5D utility scores of patients with a recent fracture attending the FLS had an average HSUV of 0.813 (0.187), 0.822 (0.180), 0.829 (0.176), 0.833 (0.180), 


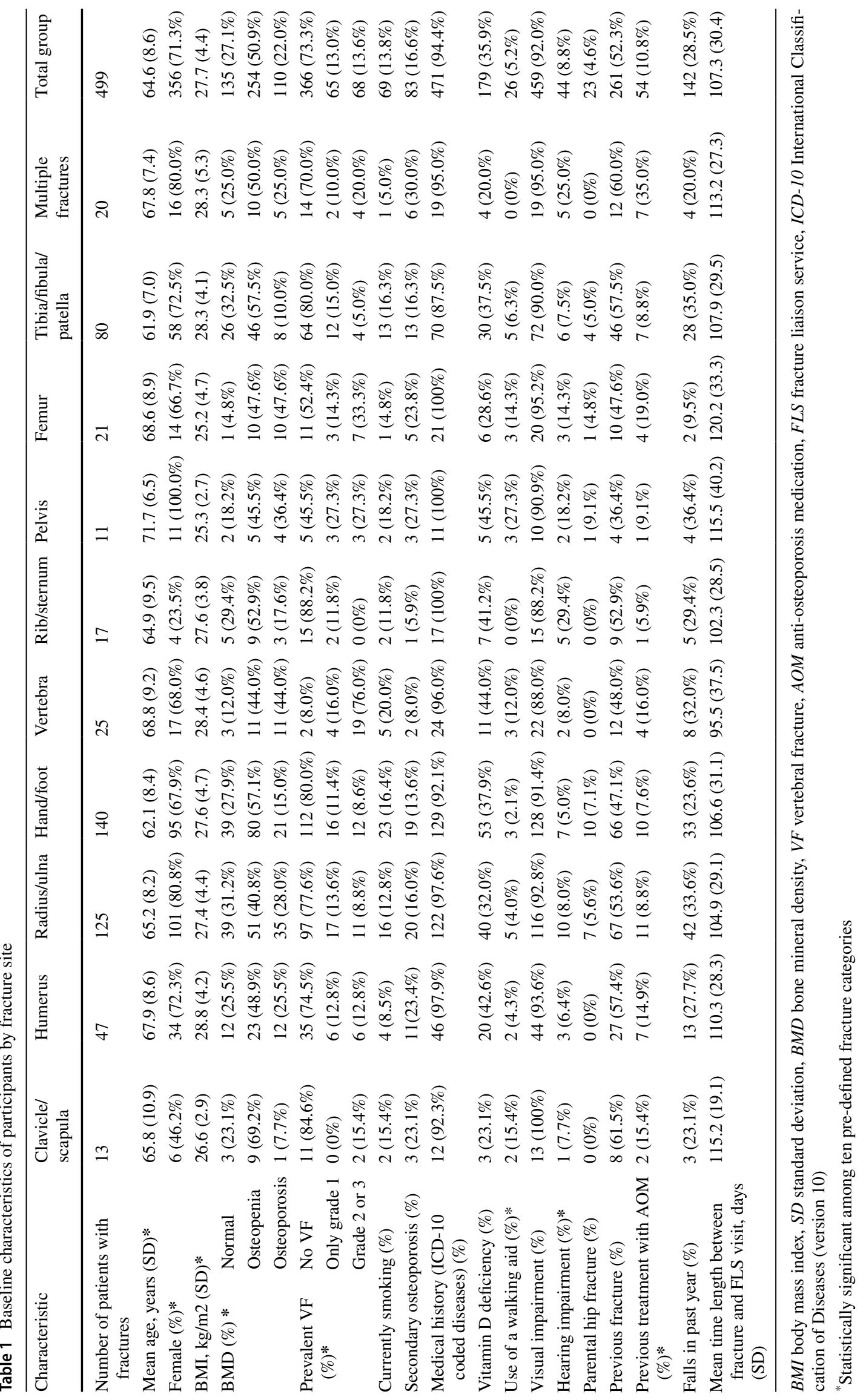


0.825 (0.196), and $0.825(0.202)$ at baseline, 3, 6, 12, 24, and 36 months, respectively (Supplement 2, Table 1). In comparison with baseline (reference), the mean HSUV of the FLS patients was slightly but significantly higher at 6 months (MD: $0.015,95 \%$ CI: $0.002-0.029 ; P=0.02$ ) and 12 months (MD: $0.018,95 \%$ CI: $0.004-0.032 ; P=0.01$ ), but not at 24 and 36 months (Table 2). When grouping patients further, significant improvement in HSUV was observed for patients with femoral, vertebral, or multiple fractures later during follow-up (i.e., at 12 and 24 months) than for patients with other fractures (significant improvement in HSUV was shown at 6 months).

Figure 1 shows the change in the HSUV over 3 years by the pre-defined ten categories based on fracture site. The linear mixed-effects model (Supplement 2, Table 2) showed that there was no association between HSUV and time regardless of the baseline fracture location, and also significant difference was not observed (interaction term: $P=0.86$ ) between these ten fracture categories in terms of the overall
HSUV change. When patients were further grouped (femo$\mathrm{ral} /$ vertebral/multiple fractures vs. other fractures), a higher yearly increase ( 0.011 vs. $<0.001$ units) was observed for patients with femoral/vertebral/multiple fractures (though which was not statistically significant).

When patients were categorized into two groups according to the length of time between index fracture and FLS visit, 258 patients had the first FLS visit $<107$ days and 241 patients $\geq 107$ days after their index fracture. As seen in Table 3 and Supplement 2 (Fig. 1), a significant increase of QoL was captured at 6, 12, and 24 months for patients who had FLS visit less than 107 days, which was not the case for the group with longer time period for FLS visit. No significant differences between these two groups were observed for age, gender, BMD, index fracture, and medical history.

With regard to the impact of subsequent fracture on EQ-5D HSUV, the analyses were performed in 479 patients of whom 50 had a subsequent fracture during follow-up. The association between EQ-5D HSUV and subsequent fracture

Table 2 The mean difference in EQ-5D HSUV between each time point and baseline by fracture location (adjusted)

\begin{tabular}{|c|c|c|c|c|c|c|c|}
\hline \multirow[t]{2}{*}{ Time point } & \multirow[t]{2}{*}{ Reference } & \multicolumn{2}{|l|}{ Total group } & \multicolumn{2}{|c|}{ Femoral/vertebral/multiple fractures } & \multicolumn{2}{|l|}{ Other fractures } \\
\hline & & $\begin{array}{l}\text { Mean difference } \\
(95 \% \mathrm{CI})\end{array}$ & $P$-value & $\begin{array}{l}\text { Mean difference } \\
(95 \% \mathrm{CI})\end{array}$ & $P$-value & $\begin{array}{l}\text { Mean difference } \\
(95 \% \mathrm{CI})\end{array}$ & $P$-value \\
\hline 3 months & Baseline & $0.008(-0.005,0.021)$ & 0.218 & $0.018(-0.021,0.057)$ & 0.352 & $0.006(-0.005,0.017)$ & 0.251 \\
\hline 6 months & & $0.015(0.002,0.029)$ & $0.024 *$ & $0.032(-0.007,0.071)$ & 0.103 & $0.013(0.001,0.025)$ & $0.039^{*}$ \\
\hline 12 months & & $0.018(0.004,0.032)$ & $0.011 *$ & $0.054(0.007,0.102)$ & $0.024 *$ & $0.012(0.001,0.025)$ & 0.056 \\
\hline 24 months & & $0.011(-0.004,0.026)$ & 0.160 & $0.046(0.002,0.090)$ & $0.041 *$ & $0.006(-0.010,0.021)$ & 0.465 \\
\hline 36 months & & $0.010(-0.005,0.026)$ & 0.193 & $0.042(-0.012,0.096)$ & 0.122 & $0.006(-0.009,0.021)$ & 0.448 \\
\hline
\end{tabular}

$C I$ confidence interval, $H S U V$ health state utility value

* Statistically significant $(P$-value $\leq 0.05)$

Adjusted: the regression analysis was adjusted for age, gender, and bone mineral density (BMD)

Fig. 1 The development of EQ-5D HSUV over time by fracture site

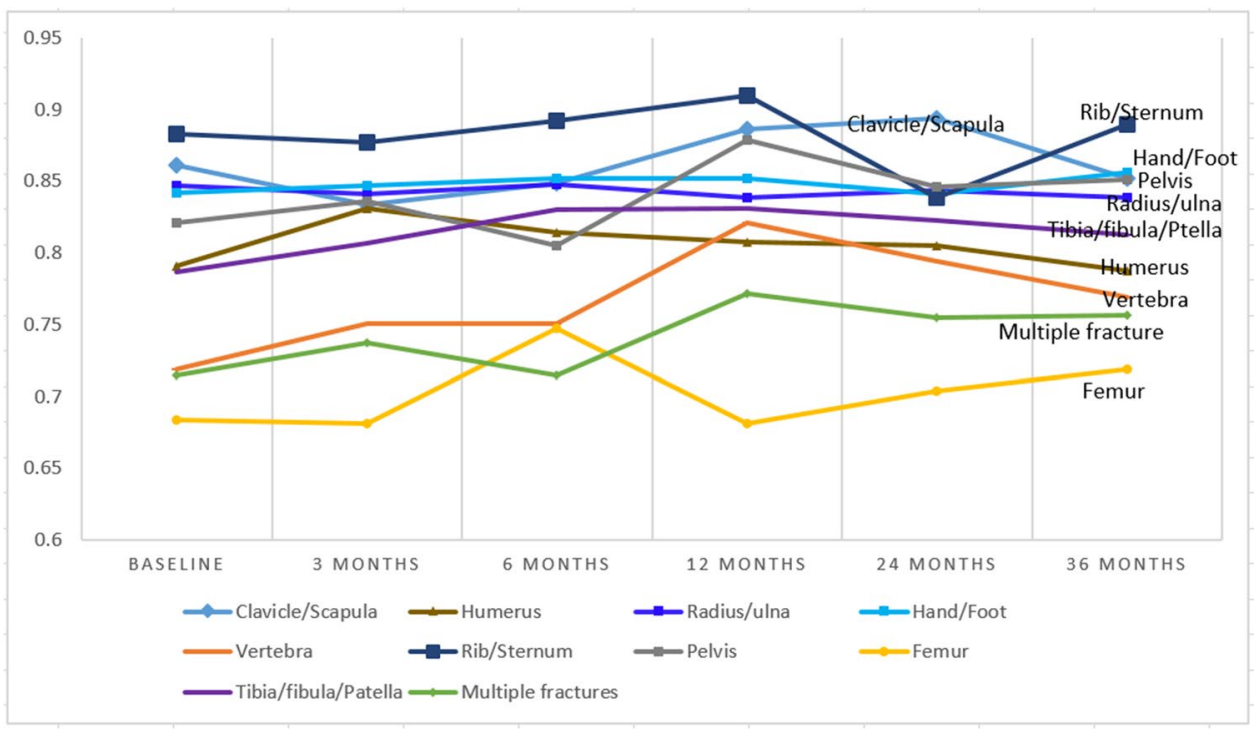


Table 3 The mean difference in EQ-5D HSUV between each time point and baseline by the time period between index fracture and FLS visit (adjusted)

\begin{tabular}{|c|c|c|c|c|c|c|}
\hline \multirow[t]{2}{*}{ Time point } & \multicolumn{3}{|c|}{ Time between fracture and baseline $<107$ days $(n=258)$} & \multicolumn{3}{|c|}{ Time between fracture and baseline $\geq 107$ days $(n=241)$} \\
\hline & Mean (SD) & $\begin{array}{l}\text { Mean difference } \\
(95 \% \mathrm{CI})\end{array}$ & $P$-value & Mean (SD) & $\begin{array}{l}\text { Mean difference } \\
(95 \% \mathrm{CI})\end{array}$ & $P$-value \\
\hline Baseline & $0.805(0.195)$ & Reference & & $0.822(0.178)$ & Reference & \\
\hline 3 months & $0.820(0.189)$ & $0.014(-0.005,0.033)$ & 0.156 & $0.823(0.170)$ & $0.002(-0.016,0.020)$ & 0.806 \\
\hline 6 months & $0.832(0.176)$ & $0.026(0.006,0.045)$ & $0.010 *$ & $0.826(0.176)$ & $0.005(-0.014,0.024)$ & 0.620 \\
\hline 12 months & $0.836(0.172)$ & $0.028(0.009,0.048)$ & $0.005^{*}$ & $0.829(0.187)$ & $0.007(-0.012,0.027)$ & 0.464 \\
\hline 24 months & $0.833(0.183)$ & $0.025(0.006,0.045)$ & $0.012 *$ & $0.817(0.208)$ & $-0.004(-0.025,0.017)$ & 0.692 \\
\hline 36 months & $0.826(0.210)$ & $0.018(-0.001,0.038)$ & 0.069 & $0.825(0.194)$ & $0.003(-0.019,0.024)$ & 0.790 \\
\hline
\end{tabular}

$C I$ confidence interval, $H S U V$ health state utility value

*Statistically significant $(P$-value $\leq 0.05)$

Adjusted: the regression analysis was adjusted for age, gender, and bone mineral density (BMD)

was displayed in Supplement 2 (Table 3). The betweensubjects interpretation indicates that the mean HSUV of patients with subsequent fracture was significantly lower ( -0.078 units) than of patients without subsequent fracture; and the within-subject interpretation indicates that a new subsequent fracture during follow-up was associated with a significant 0.078 units decrease in the mean HSUV ( 3 years). In addition, for patients had a subsequent fracture $(n=50)$, as seen in Fig. 2 and Supplement 2 (Table 4), both mean and median HSUV of post-subsequent fracture was lower than pre-subsequent fracture. The MD was - 0.078 (SD: 0.147), indicating that the suffering of a subsequent fracture resulted in significant 0.078 units decrease $(P<0.001)$ in HSUV for these patients. Moreover, when patients were subdivided into two groups, 15 patients had subsequent femoral/ vertebral/multiple fractures, and 35 had subsequent other fractures. Similar results were indicated for both groups (i.e., significantly lower HSUV for post-subsequent fracture) besides, compared to patients with subsequent other fractures, greater (but not significant) HSUV decrease was observed for patients with subsequent femoral/vertebral/ multiple fractures (MD: -0.102 vs. $-0.068, p=0.46$ ). Furthermore, when median time to subsequent fracture (364 days) was applied to compare the HSUVs in the group of patients without subsequent fracture $(n=429)$ to those with a subsequent fracture $(n=50)$, as seen in Supplement 2 (Table 5 and Fig. 2), the median and mean HSUV (in both period 1 and 2) for patients with subsequent fracture was lower than patients without subsequent fracture. For patients who suffered a subsequent fracture, the decrease in mean HSUV (MD: - 0.015) was observed in period 2 compared to period 1 whereas the increase (MD: 0.001) was captured for patients without subsequent fracture during the follow-up, both were not statistically significant.
Fig. 2 The comparison of EQ-5D HSUV before and after a subsequent fracture by the location of subsequent fracture

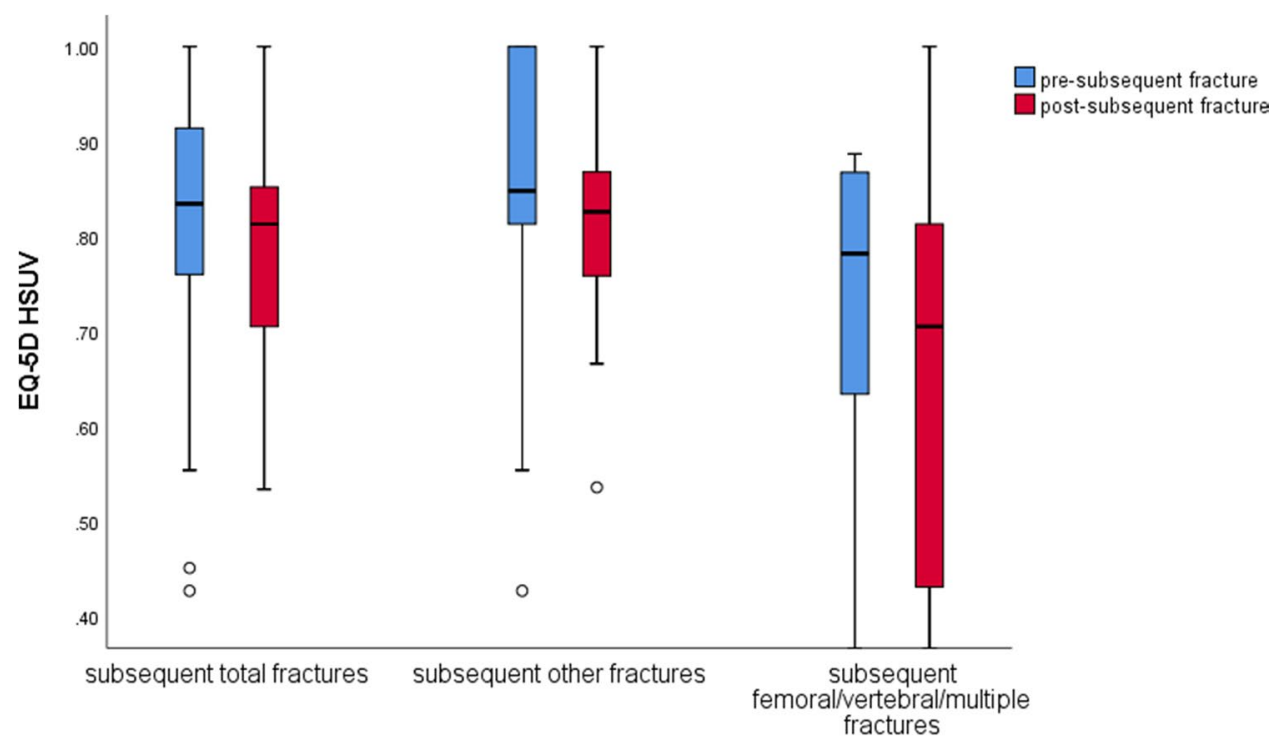

subsequent fracture 
Table 4 Factors associated with HSUV

\begin{tabular}{lllll}
\hline Covariate & EQ-5D & & SF-6D & \\
& Coefficient & $P$-value & Coefficient & $P$-value \\
Female & Exc & & -0.037 & $<0.001$ \\
Age & Exc & & Exc & \\
BMI & -0.006 & $<0.001$ & -0.004 & $<0.001$ \\
Osteopenia BMD & Exc & & Exc & \\
Osteoporotic BMD & Exc & & Exc & \\
Prevalent VFs grade 1 & Exc & & Exc & \\
Prevalent VFs grade 2 or 3 & -0.050 & 0.009 & -0.041 & 0.003 \\
Smoking & Exc & & -0.033 & 0.016 \\
Medical history (ICD-10 coded diseases) & Exc & & Exc & \\
Secondary osteoporosis & Exc & & Exc & \\
Vitamin D deficiency & Exc & & Exc & \\
Use of a walking aid & -0.279 & $<0.001$ & -0.138 & $<0.001$ \\
Visual impairment & Exc & & Exc & \\
Hearing impairment & Exc & & Exc & \\
Parental hip fracture & -0.060 & 0.047 & Exc & \\
Previous fracture & Exc & & Exc & \\
Previous treatment with AOM & -0.046 & 0.030 & Exc & \\
Falls past year & -0.038 & 0.007 & -0.028 & 0.007 \\
Falls during follow-up & Exc & & Exc & \\
Subsequent fractures during follow-up & -0.068 & $<0.001$ & -0.044 & $<0.001$ \\
\hline
\end{tabular}

$B M I$ body mass index, $B M D$ bone mineral density, $V F$ vertebral fracture, $A O M$ anti-osteoporosis medication, $I C D$-10 international classification of disease (version 10 ), $H S U V$ health state utility value

For BMD, patients with normal BMD was the reference, for prevalent VFs, patients without VF was the reference

Exc $=$ covariate was excluded in stepwise selection process

\section{SF-6D health utility (sensitivity analysis)}

The above-mentioned analyses were also conducted using SF-6D HSUV; the statistical results are shown in Supplement 3. In comparison with baseline HSUV, a statistically significant increase was also captured at 6 months (Supplement 3, Table 2). Besides, the longitudinal regression also indicated that the change in HSUV over time was not significant for the total cohort, and the course of SF-6D HSUV was also not significantly different between pre-defined ten fracture categories. In addition, when patients were categorized into two groups according to the length of time between index fracture and FLS visit, compared to baseline HSUV, a significant increase was also captured at 6 and 12 months for patients had FLS visit less than 107 days, which was not the case for the group with longer time period for FLS visit (Supplement 3, Table 3). Moreover, for patients who had a subsequent fracture, both mean and median HSUV of postsubsequent fracture was also lower than pre-subsequent fracture, and the suffering of a subsequent fracture resulted in significant decrease in HSUV for these patients. The results remained when patients were subdivided by subsequent fracture location (Supplement 3, Table 4 and Fig. 2). Furthermore, when median time to subsequent fracture (364 days) was applied to compared the HSUVs in the group of patients without subsequent fracture to those with a subsequent fracture, the median and mean HSUV (in both period 1 and 2) for patients with subsequent fracture was also lower than patients without subsequent fracture (Supplement 3, Fig. 3).

\section{Factors associated with EQ-5D and SF-6D HSUV}

The stepwise regression analysis (Table 4) indicated the effect of covariates on patients' mean HSUV over 3 years (six time point). BMI, use of a walking aid, AOM treatment, previous falls, prevalent VF (grade 2 or 3), and subsequent fracture were identified as factors associated with mean EQ-5D HSUV. With regard to time-varying covariates, a negative predictive relationship between subsequent fracture and HSUV was indicated (the between- and within-subject interpretations were similar to previously described). As for time-invariant covariates, patients using a walking aid, taking AOM, and having experienced previous falls had significantly lower HSUV in comparison to their counterparts. To investigate the association between prevalent vertebral fractures (VFs) and HSUV, patients without prevalent VFs were set as the reference; it can be seen from Table 4 that only prevalent VFs grade 2 or 3 was identified as a 
factor associated with HSUV. The effect size can be interpreted that patients with prevalent VFs grade 2 or $3 \mathrm{had}$ significantly 0.050 units lower EQ-5D HSUV on average (mean HSUV of 3 years) than patients without prevalent VFs. Other covariates like age and smoking were excluded through the process of backward stepwise elimination based on $P$-value, but we found that the increasing in patients' age was associated with decreasing in mean HSUV (1-year age increase was associated with 0.001 units decrease in mean HSUV), and smokers had (0.030 units) lower HSUV than non-smokers.

In addition to the above-mentioned factors (by EQ-5D), SF-6D indicated another two factors (gender and smoking). In comparison with males, females had a significantly lower HSUV on average, and smokers reported significantly lower utility in comparison with non-smokers.

\section{Discussion}

This study, to our knowledge, is the first longitudinal study using prospective data over 3 years, with the objective of estimating the HRQoL of patients following visits to a FLS because of a recent fracture. With regard to EQ-5D HSUV, no significant change was captured over 3 years, although a small but statistically significant improvement was observed at 6 and 12 months in comparison with baseline HSUV. This short-term improvement is more likely due to natural healing of the fracture rather than the effect of attending an FLS or having a fracture risk evaluation at the FLS. When patients were stratified by baseline fracture (femoral/vertebral/multiple fractures vs. other fractures), our results remained (i.e., there was no significant overall change). We did not find any previous study investigating the long-term HRQoL of patients attending a FLS. In contrast, two studies [14, 15] compared the HSUV of patients with a recent fracture before and after the introduction of a FLS; no significant difference between two groups was identified at the 6 or 12 months follow-up.

The primary potential reason for a non-significant change in HSUV over 3 years is that the patients included in our study attended the FLS 3-4 months after their index fracture; thus, their HRQoL might have already improved through natural fracture recovery and/or through treatment in the emergency department before attending the FLS, resulting in non-striking change after attending FLS. This was also found in the ICUROS studies [9, 11, 12]: although substantial loss of HRQoL was captured in the short term after fracture, patients' HRQoL was largely improved at 4 months. Except for ICUROS studies, the improvement was also identified in patients who did not attend a FLS in recent studies [14, 15], which can also be attributable to natural healing (recovery) of the fracture. The second potential reason for lack of significant change may be selection bias; it is likely that patients with more severe fractures, older patients, or patients who were hospitalized did not attend the FLS. Furthermore, approximately half of FLS attenders did not consent to participate in this study. In this study, the average baseline HSUV was 0.81 , which is a bit lower but still comparable to the HSUV of community-dwelling Dutch residents aged 65 years and older as reported by Mangen et al. [23]. Therefore, our study included relatively healthy patients, which may have resulted in a relatively good HRQOL after the index fracture, without a significant change over time.

Additionally, the greater increase in QOL in patients with a shorter time period ( $<107$ days) between index fracture and FLS visit may be attributed to the earlier stage of the fracture healing process, and by the timely treatment from FLS clinic compared to patients with a longer time period to FLS visit.

Of note, although patients' baseline HRQoL was measured 3-4 months after their index fracture, patients with femoral, vertebral, or multiple fractures still had a lower HSUV in comparison with patients with other fractures. Also, significant improvement in HRQoL takes a longer time to capture (baseline HSUV as the reference) as reported in our study. Fisher et al. [24] assessed the timeline of functional recovery after hip fracture in seniors (aged 65 years and older) and reported that objective functional recovery (lower extremity function) was largely complete in the first 6 months, whereas subjective recovery (HRQoL) improved up to 9 months after hip fracture. In addition, we also identified that the number of baseline fractures impacted patients' HRQoL. Patients with multiple fractures had a significantly lower HSUV in comparison with patients with only one index fracture such as clavicle/scapula, radius/ulna, hand/ foot, rib/sternum fracture. This finding is supported by a previous study showing that patients with multiple clinical fractures would experience an additive effect, resulting in disability similar to a single hip or vertebral fracture [25] which is in line with our finding that the absolute average HSUV (over 3 years) difference between femoral, vertebral, and multiple fractures was not significant.

Suffering a subsequent fracture was associated with a decrease in HSUV; this finding is supported by several previous studies [26-28] reporting that subsequent fractures have a significantly negative impact on the QoL, greater loss of function, and increased mortality. However, whether the effect of FLS is larger immediately after subsequent fracture, and whether the cost-effectiveness of FLS is somewhat better, it remains unknown based on our data and recently published studies. Furthermore, to accurately estimate the effect of FLS on QoL is even difficult given QoL is determined by multiple factors such as lifestyle, aging, and comorbidities; however, it would be interesting for future studies. Also, the 
presence of a moderate or severe prevalent VF was associated with a lower HSUV, which is in line with recent findings of Shah et al. [29]. Besides, patients who previously received treatment with AOM reported lower utility in comparison with their counterparts. The lower HSUV might be explained by two aspects: first, since the indication for treatment (according to the Dutch guideline) is osteoporosis and/ or a moderate or severe VF, patients' awareness of having osteoporosis and/or VFs and increased future fracture risk might result in over cautiousness and limiting daily activities; second, the potential side effects of oral AOM including bone, joint, or muscle pain, as well as nausea, difficulty swallowing, and heartburn might affect patients' HRQoL, and the rare side effects such as osteonecrosis of the jaw and atypical femoral fracture actually scare many patients away from taking AOM, with the result that suboptimal persistence and adherence lead to an increased rate of fracture and to worse HRQoL [30]. Moreover, patients' HSUVs following a fracture are clearly negatively associated with high BMI since excessive body fat produces inflammatory cytokines which may stimulate bone resorption and reduce bone strength [31]. Several recent studies reported that both low and high BMI are risk factors for fragility fracture. The study of Yan et al. [32] investigated the relationships of BMI with HRQoL in adults 65 years and older and revealed that compared with normal-weight people, both underweight and obese older adults reported impaired QoL, particularly worse physical functioning and physical well-being. Furthermore, some previous studies identified that gender $[13,16]$ and previous fracture [9] are important factors; these were not captured by EQ-5D in our study.

In a sensitivity analysis using another HRQoL measure, namely the SF-6D, comparable results were identified and our main message remained, though there were small differences. For example, the mean SF-6D HSUV was generally lower than the EQ-5D HSUV, and only SF-6D indicated that the course of HSUV for patients with hand/foot fracture was significant. Besides, when the median time to subsequent fracture (364 days) was applied to compare the HSUVs patients without subsequent fracture to those with a subsequent fracture, a higher median EQ-5D HSUV was captured in period 2 for both groups, however, which was only observed for patients without subsequent fracture by SF-6D HSUV. These discrepancies between the two instruments might result from differences in the content of the descriptive systems and in the variation of scoring algorithms [33]; a further head-to-head comparison will be conducted to explore the equivalency of EQ-5D-5L and SF-6D crosssectionally, including scoring distribution, domain content, and longitudinal validity.

This study has some limitations. First, relatively healthy patients were included in this study (selection bias), so the generalizability of the findings of this study to patients with a fracture could therefore be questionable. Second, our patients attended the FLS approximately 3-4 months after their index fracture; we therefore lacked HRQoL data immediately after fracture, which might limit our capturing an overall significant change of HRQoL in the first several weeks of recovery from a fracture. Finally, 18 patients scored an item of EQ-5D twice; we calculated their two utility scores and used the average value for further analyses, which might affect the results.

\section{Conclusion}

In patients at the FLS, subsequent fracture, previous treatment with AOM, a prevalent VF (grade 2 or 3), use of a walking aid, previous falls, and higher BMI were negatively associated with EQ-5D HSUV. The change in HSUV over the total course of 3 years was not statistically significant, although significant improvements were observed at 6- and 12-month time points compared to baseline. There was no significant difference in the course of HSUV between the pre-defined ten fracture categories. A significant increase in HSUV was only captured for patients had shorter time period ( $<107$ days) between FLS visit and their index fracture. Suffering a subsequent fracture was associated with significant QoL loss.

Supplementary Information The online version contains supplementary material available at https://doi.org/10.1007/s00198-021-06204-x.

Funding Nannan Li is funded by the China Scholarship Council (grant number 201909110080)

Data availability All data analyzed as part of this study are included in this published article (and its supplementary information files).

\section{Declarations}

Conflicts of interest Joop P. van den Bergh has received research funding from Amgen, and UCB; Mickaël Hiligsmann has received research grants through institution from Amgen, Radius Health, and ViiV, consulting fee from UCB, and lecture fee from Mylan Pharmaceuticals; Annelies Boonen, Sandrine P.G. Bours, Caroline E. Wyers, Sander MJ van Kuijk, Lisanne Vranken, and Marsha M. van Oostwaard declare that they have no conflict of interest.

Open Access This article is licensed under a Creative Commons Attribution-NonCommercial 4.0 International License, which permits any non-commercial use, sharing, adaptation, distribution and reproduction in any medium or format, as long as you give appropriate credit to the original author(s) and the source, provide a link to the Creative Commons licence, and indicate if changes were made. The images or other third party material in this article are included in the article's Creative Commons licence, unless indicated otherwise in a credit line to the material. If material is not included in the article's Creative Commons licence and your intended use is not permitted by statutory regulation or exceeds the permitted use, you will need to obtain permission 
directly from the copyright holder. To view a copy of this licence, visit http://creativecommons.org/licenses/by-nc/4.0/.

\section{References}

1. Sozen T, Ozisik L, Calik BN (2017) An overview and management of osteoporosis. Eur J Rheumatol 4(1):46-56. https://doi. org/10.5152/eurjrheum.2016.048

2. Lötters FJB, van den Bergh JP, de Vries F, Rutten-van Mölken MPMH (2016) Current and future incidence and costs of osteoporosis-related fractures in The Netherlands: combining claims data with BMD measurements. Calcif Tissue Int 98(3):235-243. https://doi.org/10.1007/s00223-015-0089-Z

3. Van Geel TACM, Huntjens KMB, Van Den Bergh JPW, Dinant GJ, Geusens PP (2010) Timing of subsequent fractures after an initial fracture. Curr Osteoporos Rep 8(3):118-122. https://doi. org/10.1007/s11914-010-0023-2

4. Tran T, Bliuc D, Hansen L et al (2018) Persistence of excess mortality following individual nonhip fractures: a relative survival analysis. J Clin Endocrinol Metab 103(9):3205-3214. https://doi.org/10.1210/jc.2017-02656

5. McLellan AR, Gallacher SJ, Fraser M, McQuillian C (2003) The fracture liaison service: success of a program for the evaluation and management of patients with osteoporotic fracture. Osteoporos Int 14(12):1028-1034. https://doi.org/10.1007/ s00198-003-1507-z

6. Åkesson K, Marsh D, Mitchell PJ et al (2013) Capture the fracture: a best practice framework and global campaign to break the fragility fracture cycle. Osteoporos Int 24(8):2135-2152. https://doi.org/10.1007/s00198-013-2348-Z

7. Lems WF, Dreinhöfer KE, Bischoff-Ferrari H et al (2017) EULAR/EFORT recommendations for management of patients older than 50 years with a fragility fracture and prevention of subsequent fractures. Ann Rheum Dis 76(5):802-810. https:// doi.org/10.1136/annrheumdis-2016-210289

8. Eisman JA, Bogoch ER, Dell R et al (2012) Making the first fracture the last fracture: ASBMR task force report on secondary fracture prevention. J Bone Miner Res 27(10):2039-2046. https://doi.org/10.1002/jbmr.1698

9. Svedbom A, Borgström F, Hernlund E et al (2018) Quality of life after hip, vertebral, and distal forearm fragility fractures measured using the EQ-5D-3L, EQ-VAS, and time-trade-off: results from the ICUROS. Qual Life Res 27(3):707-716. https:// doi.org/10.1007/s11136-017-1748-5

10. Wolowacz SE, Briggs A, Belozeroff V et al (2016) Estimating health-state utility for economic models in clinical studies: an ISPOR good research practices task force report. Value Heal 19(6):704-719. https://doi.org/10.1016/j.jval.2016.06.001

11. Borgström F, Lekander I, Ivergård M et al (2013) The International Costs and Utilities Related to Osteoporotic Fractures Study (ICUROS) - quality of life during the first 4 months after fracture. Osteoporos Int 24(3):811-823. https://doi.org/10.1007/ s00198-012-2240-2

12. Lesnyak O, Svedbom A, Belova K, et al. (2020) Quality of life after fragility fracture in the Russian Federation: results from the Russian arm of the International Cost and Utility Related to Osteoporotic Fractures Study (ICUROS). Arch Osteoporos 15(1). https://doi.org/10.1007/s11657-020-0699-6

13. Si L, Winzenberg TM, De Graaff B, Palmer AJ (2014) A systematic review and meta-analysis of utility-based quality of life for osteoporosis-related conditions. Osteoporos Int 25(8):19871997. https://doi.org/10.1007/s00198-014-2636-2

14. Singh S, Whitehurst DG, Funnell L, et al. (2019) Breaking the cycle of recurrent fracture: implementing the first fracture liaison service (FLS) in British Columbia, Canada. Arch Osteoporos 14(1). https://doi.org/10.1007/s11657-019-0662-6

15. Inderjeeth CA, Raymond WD, Briggs AM, Geelhoed E, Oldham D, Mountain D (2018) Implementation of the Western Australian Osteoporosis Model of Care: a fracture liaison service utilising emergency department information systems to identify patients with fragility fracture to improve current practice and reduce re-fracture rates: a 12-. Osteoporos Int 29(8):1759-1770. https://doi.org/10.1007/s00198-018-4526-5

16. Adachi JD, Ioannidis G, Berger C et al (2001) The influence of osteoporotic fractures on health-related quality of life in community-dwelling men and women across Canada. Osteoporos Int 12(11):903-908. https://doi.org/10.1007/s001980170017

17. Jun HJ, Kim KJ, Lee JS, Yang SH (2015) Association between osteoporotic fractures and quality of life based on the Korean Community Health Survey of 2010. J Phys Ther Sci 27(10):3325-3328. https://doi.org/10.1589/jpts.27.3325

18. Vranken L, Wyers CE, van Rietbergen B et al (2019) The association between prevalent vertebral fractures and bone quality of the distal radius and distal tibia as measured with HR-pQCT in postmenopausal women with a recent non-vertebral fracture at the Fracture Liaison Service. Osteoporos Int 30(9):1789-1797. https://doi.org/10.1007/s00198-019-05081-9

19. Kanis JA, Adachi JD, Cooper C et al (2013) Standardising the descriptive epidemiology of osteoporosis: recommendations from the Epidemiology and Quality of Life Working Group of IOF. Osteoporos Int 24(11):2763-2764. https://doi.org/10.1007/ s00198-013-2413-7

20. Genant HK, Wu CY, van Kuijk C, Nevitt MC (1993) Vertebral fracture assessment using a semiquantitative technique. J Bone Miner Res 8(9):1137-1148. https://doi.org/10.1002/jbmr.56500 80915

21. Versteegh M, Vermeulen MK, Evers MAAS, de Wit GA, Prenger R, Stolk AE (2016) Dutch tariff for the five-level version of EQ-5D. Value Heal 19(4):343-352. https://doi.org/10. 1016/j.jval.2016.01.003

22. Brazier J, Roberts J, Deverill M (2002) The estimation of a preference-based measure of health from the SF-36. J Health Econ 21(2):271-292. https://doi.org/10.1016/S0167-6296(01) 00130-8

23. Mangen MJJ, Bolkenbaas M, Huijts SM, van Werkhoven CH, Bonten MJM, de Wit GA (2017) Quality of life in community-dwelling Dutch elderly measured by EQ-5D-3L. Health Qual Life Outcomes 15(1):1-6. https://doi.org/10.1186/ s12955-016-0577-5

24. Fischer K, Trombik M, Freystätter G, Egli A, Theiler R, Bischoff-Ferrari HA (2019) Timeline of functional recovery after hip fracture in seniors aged 65 and older: a prospective observational analysis. Osteoporos Int 30(7):1371-1381. https://doi. org/10.1007/s00198-019-04944-5

25. Adachi JD, Adami S, Gehlbach S et al (2010) Impact of prevalent fractures on quality of life: baseline results from the global longitudinal study of osteoporosis in women. Mayo Clin Proc 85(9):806-813. https://doi.org/10.4065/mcp.2010.0082

26. Jinbayashi H, Aoyagi K, Ross PD, Ito M, Shindo H, Takemoto $\mathrm{T}$ (2002) Prevalence of vertebral deformity and its associations with physical impairment among Japanese women: the HizenOshima study. Osteoporos Int 13(9):723-730. https://doi.org/ 10.1007/s001980200099

27. Bliuc D, Alarkawi D, Nguyen TV, Eisman JA, Center JR (2015) Risk of subsequent fractures and mortality in elderly women and men with fragility fractures with and without osteoporotic bone density: the dubbo osteoporosis epidemiology study. $\mathrm{J}$ Bone Miner Res 30(4):637-646. https://doi.org/10.1002/jbmr. 2393 
28. Inose H, Kato T, Ichimura S, et al. (2021) Risk factors for subsequent vertebral fracture after acute osteoporotic vertebral fractures. Eur Spine J (0123456789). https://doi.org/10.1007/ s00586-021-06741-3

29. Shah A, Wu F, Jones G, Cicuttini F, Toh LS, Laslett LL. The association between incident vertebral deformities, healthrelated quality of life and functional impairment : a 10 . 7-year cohort study. Published online 2021.

30. Yeam CT, Chia S, Tan HCC, Kwan YH, Fong W, Seng JJB (2018) A systematic review of factors affecting medication adherence among patients with osteoporosis. Osteoporos Int 29(12):2623-2637. https://doi.org/10.1007/s00198-018-4759-3

31 Gonnelli S, Caffarelli C, Nuti R (2014) Obesity and fracture risk. Clin Cases Miner Bone Metab 11(1):9-14. https://doi.org/ 10.11138/ccmbm/2014.11.1.009
32. Yan LL, Daviglus ML, Liu K et al (2004) BMI and healthrelated quality of life in adults 65 years and older. Obes Res 12(1):69-76. https://doi.org/10.1038/oby.2004.10

33 Sayah F Al, Qiu W, Xie F, Johnson JA. Comparative performance of the EQ-5D-5L and SF-6D index scores in adults with type 2 diabetes. Qual Life Res. 2017;26(8):2057-2066. https://doi.org/ $10.1007 / \mathrm{s} 11136-017-1559-8$

Publisher's note Springer Nature remains neutral with regard to jurisdictional claims in published maps and institutional affiliations. 